\title{
NOTES
}

\section{Finding of Columnar Phases of a Thermotropic-Liquid Crystalline Polypeptide}

\author{
Eisaku IrzuKa and Shuji IIDA* \\ Department of Functional Polymer Science, Faculty of Textile Science \\ and Technology, Shinshu University, Ueda, Nagano 386, Japan.
}

(Received November 11, 1989)

KEY WORDS Polypeptide / Thermotropic Liquid Crystal / Molecular
Arrangement / Columnar Phase / Rectangular Packing / Hexagonal Packing /

Poly(L-glutamate) esters with long alkyl side chains containing a mesogenic aromatic segment at the terminal position are $\alpha$-helical ${ }^{1}$ and form thermotropic liquid crystals when the side chains melt upon heating. ${ }^{2}$ Arrangement of polymer helices both in crystalline and liquid-crystalline states was investigated using the specimen with side chains having 6 carbons in the alkyl segment and 1 benzene group in the mesogenic segment. The abbreviation for this specimen is $\mathrm{R}^{\prime \prime} 6$ according to the previous paper. ${ }^{2}$ The degree of polymerization (DP) of $\mathrm{R}^{\prime \prime} 6$ is 940 and the degree of substitution (DS) being $98 \%$. This specimen undergoes the crystal-to-liquid crystal transition at about $27^{\circ} \mathrm{C}$.

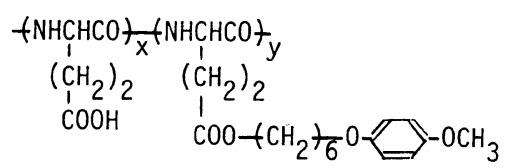

The specimen was rolled with a spatula to make a thin rod of diameter slightly less than $1 \mathrm{~mm}$, put in a 1-mm X-ray glass capillary, heated for $3 \mathrm{~h}$. at a liquid-crystalline temperature of $160^{\circ} \mathrm{C}$ in an oil bath, and cooled naturally to room temperature by turning off the bath to assure the full growth of a crystalline structure.
As may be seen in Figure 1, the specimen shows orientation in the direction of the rod axis after being rolled (pattern (a)) and the degree of orientation was enhanced markedly by the annealing treatment (pattern (b)). This indicates that in liquid crystalline state the polymer helices further align themselves parallel with each other and parallel to the axis of the capillary. The rolling of the specimen prior to annealing treatment is to help the polymer helices better orient in liquid-crystalline state; the orientation phenomenon was observed even without such a pretreatment though only slightly.

$\mathrm{X}$-Ray analysis showed that the packing of the polymer helices in the oriented specimen was rectangular as shown in Table I. The cross section of the unit cell has dimensions, $a=45.12 \AA$ and $b=13.16 \AA$. The number of the polymer helix in the unit cell is 2 , and the calculated density of the polymer crystal is $1.226 \mathrm{~g} \mathrm{~cm}^{-3}$, whereas the density as measured by the submerged-float method in aqueous $\mathrm{KBr}$ solution is $1.220 \mathrm{~g} \mathrm{~cm}^{-3}$. Considering that the crystalline structure of the specimen is possibly not perfect, these two density values would agree with each other very well.

The distance between the helical axis of the $\mathrm{R}^{\prime \prime} 6$ molecule and the terminal hydrogen atom

\footnotetext{
* Present address: Chikumagawa Factory, TDK, Saku, Nagano 385, Japan.
} 

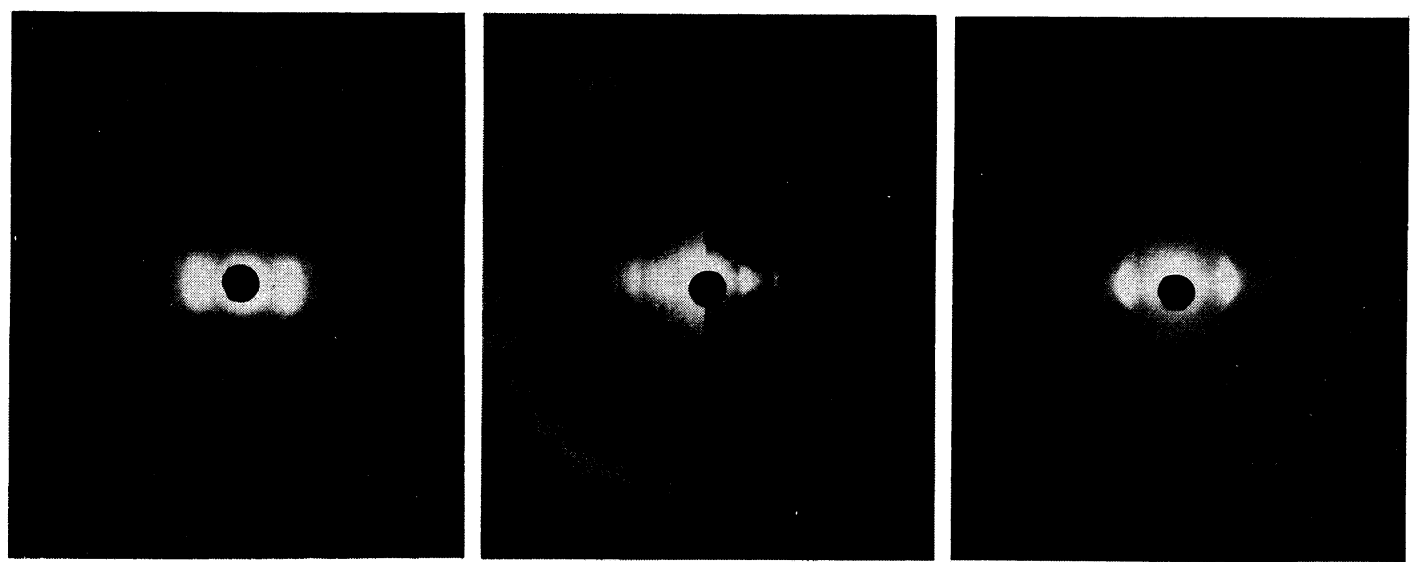

Figure 1. X-Ray diffraction patterns of $\mathrm{R}^{\prime \prime} 6 . \mathrm{Cu}-K_{\alpha}$ radiation, $D=9.3 \mathrm{~cm} ; D$ is the distance from the specimen to the photographic film. The X-ray capillary was placed vertical. The photograph was taken at room temperature (a) after the specimen had been rolled, or (b) after it had been rolled and heated for $3 \mathrm{~h}$ at $160^{\circ} \mathrm{C}$, or taken (c) at $60^{\circ} \mathrm{C}$ after it had been rolled, heated for $3 \mathrm{~h}$ at $160^{\circ} \mathrm{C}$ and cooled to room temperature.

Table I. Equatorial spacings in the $\mathrm{X}$-ray diffraction photograph of oriented $\mathrm{R}^{\prime \prime} 6$ in crystalline state

\begin{tabular}{ccc}
\hline Lattice plane & $d_{\text {obs. }} / \AA$ & $d_{\text {calc. }} / \AA$ \\
\hline$d_{1}(200)$ & 22.56 & 22.56 \\
$d_{2}(110)$ & 12.63 & 12.63 \\
$d_{3}(210)$ & 11.38 & 11.37 \\
$d_{4}(310)$ & 9.87 & 9.90 \\
$d_{5}(600)$ & 7.48 & 7.52 \\
$(510)$ & 6.31 & 7.44 \\
$d_{6}(220)$ & & 6.32 \\
$d_{7}(420)$ & 5.67 & 5.68 \\
$(800)$ & & 5.64 \\
\hline
\end{tabular}



$a=45.12 \AA$
$b=13.16 \AA$ of its fully extended side chains is $24.2 \AA$. (This was obtained by adding the known distance for PBLG, ${ }^{3} 14.1 \AA$, to the length of the remainder of the side chain assuming that the bond lengths were $1.53 \AA$ and the bond angles were $112^{\circ}$.) The value of the distance thus estimated strongly suggests that the side chains are extended perpendicular to the polymer helix and parallel to the $a$-direction of the unit cell.

In a liquid-crystalline temperature of $60^{\circ} \mathrm{C}$, $\mathrm{X}$-ray diffraction from the oriented $\mathrm{R}^{\prime \prime} 6$ reasonably became broad and diminished in number (Figure 1, pattern (c)). The spacing corresponding to the innermost reflection fell off from $22.56 \AA$ to $17 \AA$. This can be elucidated by assuming that the packing of the polymer helices turned to hexagonal; the innermost reflection is now due to the 110 lattice plane instead of the 200 lattice plane. The cross section of the unit cell that agrees with the observed density of $1.180 \mathrm{~g} \mathrm{~cm}^{-3}$ has dimensions, $a^{\prime}=b^{\prime}=19.3 \AA$ and $\gamma=124^{\circ}$. The packing 
of the polymer helices is therefore not accurately hexagonal; however, the deviation is very small. The deviation observed is exactly same as that observed in the hexagonal packing of $\mathrm{PBLG}^{3}$ in crystalline state.

These two modes of the packing of the polymer helices, one in crystalline state and the other in liquid-crystalline state, are considered to fall under the category of columnar phase. The hexagonal packing is first formed due to some wall effect of the capillary to orient the polymer helices parallel to its axis. It is known that a liquid-crystalline racemic solution containing equal proportions of PBLG and PBDG shows regular orientation in the direction of the axis of a $1.0-\mathrm{mm}$ or $0.5-\mathrm{mm}$ capillary. ${ }^{4}$ The molten alkyl side chains of the polymer helices of $\mathrm{R}^{\prime \prime} 6$ would act as the solvent as in the case of such a lyotropic liquid crystal to help the polymer helices orient in a similar way.

Reorientation of the polymer helices becomes easier at a higher liquid-crystalline temperature where the viscosity of the polymer system is low. The annealing temperature, however, cannot be set up beyond a certain critical temperature, above which the specimen starts to degrade. Therefore, the rolling pretreatment is necessary to help the polymer helices better make the spontaneous orientation.

In addition to $\mathrm{R}^{\prime \prime} 6$, a poly(L-glutamate) ester of a similar kind, of which the number of carbons in the side chain is 10 , and a PBLG ester with long alkyl side chains, ${ }^{5}$ each of which contains 16 carbons, were also tested. The abbreviation for the former is $\mathrm{R}^{\prime \prime} 10$ and that for the latter being R16. ${ }^{6}$ The DP is 940 in $\mathrm{R}^{\prime \prime} 10$ and 60 in $\mathrm{R} 16$, while the DS is $97 \%$ in $R^{\prime \prime} 10$ and $100 \%$ in $R 16 . R^{\prime \prime} 10$ becomes liquid crystalline at a higher temperature than $R^{\prime \prime} 6$ (about $43^{\circ} \mathrm{C}$ ). ${ }^{2} \mathrm{R} 16$ becomes liquid crystalline at $42^{\circ} \mathrm{C}$, gets more fluent with temperature, and turns to isotropic at $160^{\circ} \mathrm{C}^{7}$ Both of the specimens did not show the spontaneous orientation in a capillary probably due to the lack of fluidity for $\mathrm{R}^{\prime \prime} 10$ and possibly the lack of polymer length in comparison with the diameter of the capillary for R16.

In conclusion, the specimen $\mathrm{R}^{\prime \prime} 6$ after making spontaneous orientation in a thin $\mathrm{X}$-ray glass capillary is in columnar phase both in crystalline and liquid-crystalline states. The molecular packing is rectangular in crystalline state and hexagonal in liquid-crystalline state to better pack the polymer helices with alkyl side chains which are stiff, extending perpendicular to the polymer helix in the former state and flexible in the latter state.

Acknowledgment. We wish to thank Drs. K. Hanabusa and H. Shirai of our Department for the donation of the polypeptide specimens used in this study and Dr. K. Ohta, also of our Department, for his useful suggestions in the analysis of the X-ray data obtained.

\section{REFERENCES}

1. K. Hanabusa, K. Yanagisawa, J. Higashi, H. Shirai, T. Hayakawa, and N. Hojo, J. Polym. Sci., Polym. Chem. Ed., in press.

2. E. Iizuka and S. Iida, in "Dynamic Behavior of Macromolecules, Colloids, Liquid Crystals and Biological Systems by Optical and Electro-Optical Methods," H. Watanabe, Ed., Hirokawa Pub. Co., Tokyo, 1988, p 383.

3. C. H. Bamford, A. Elliott, and W. E. Hanby, "Synthetic Polypeptides," Academic Press, New York, 1956, p 263.

4. C. Robinson, J. C. Ward, and R. B. Beevers, Disc. Faraday Soc., 25, 29 (1958).

5. K. Hanabusa, M. Sato, H. Shirai, K. Takemoto, and E. Iizuka, J. Polym. Sci., Polym. Lett. Ed., 22, 559 (1984).

6. E. Iizuka, K. Abe, K. Hanabusa, and H. Shirai, in "Current Topics in Polymer Science," Vol. 1, R. M. Ottenbrite, L. A. Utracki, and S. Inoue, Eds., Hanser Publishers, New York, 1987, p 235.

7. E. Iizuka, S. Inoue, K. Hanabusa, and H. Shirai, Mol. Cryst., Liq. Cryst., 149, 61 (1987). 